\title{
IPv6-in-IPv4 tunnel discovery: methods and experimental results
}

\author{
Lorenzo Colitti, Student Member, IEEE, Giuseppe Di Battista, and Maurizio Patrignani
}

\begin{abstract}
Tunnels are widely used to improve security and to expand networks without having to deploy native infrastructure. They play an important role in the migration to IPv6, which relies on IPv6-in-IPv4 tunnels where native connectivity is not available; however, tunnels offer lower performance and are less reliable than native links. In this paper we introduce a number of techniques to detect, and collect information about, IPv6-in-IPv4 tunnels, and show how a known tunnel can be used as a "vantage point" to launch third-party tunnel-discovery explorations, scaling up the discovery process. We describe our Tunneltrace tool, which implements the proposed techniques, and validate them by means of a wide experimentation on the 6bone tunneled network, on native networks in Italy, the Netherlands, and Japan, and through the test boxes deployed worldwide by the RIPE NCC as part of the Test Traffic Measurements Service. We assess to what extent 6bone registry information is coherent with the actual network topology, and we provide the first experimental results on the current distribution of IPv6-in-IPv4 tunnels in the Internet, showing that even "native" networks reach more than $60 \%$ of all IPv6 prefixes through tunnels. Furthermore, we provide historical data on the migration to native IPv6, showing that the impact of tunnels in the IPv6 Internet did not significantly decrease over a 6-month period. Finally, we briefly touch on the security issues posed by IPv6-in-IPv4 tunnels, discussing possible threats and countermeasures.
\end{abstract}

Index Terms- Tunnels, IPv6, Tunnel Discovery, IPv6 Topology Discovery, IPv4 to IPv6 Transition

\section{INTRODUCTION}

$\mathbf{T}$ UNNELLING consists in the encapsulation of the packets of a network protocol within the packets of a second network protocol, such that the former regards the latter as its datalink layer [1]. Because of the flexibility it provides (any protocol can be transported, including the encapsulating protocol itself), tunnelling is widely used both to expand networks without having to deploy native infrastructure and to improve security. Examples of the former include IPX-inIP encapsulation [2], IPv6-in-IP encapsulation [3] and IP-in-IP encapsulation [4]; examples of the latter include IPsec [5] and virtual private networks [6]. The IPv6 specifications define several types of IPv6-in-IPv4 tunnels, including configured tunnels and automatic tunnels [3], 6to4 [7], ISATAP [8], and Teredo [9]; IPv6 may also use GRE tunnels over IPv4 [10]. Our results suggest that tunnels are very common in

The authors are with Roma Tre University. Work partially supported by European Commission: 6NET (IST-2001-32603) and Fet Open project COSIN (IST-2001-33555); by "Progetto ALINWEB: Algoritmica per Internet e per il Web", MIUR Programmi di Ricerca Scientifica di Rilevante Interesse Nazionale; and by European Commission - Fet Open project DELIS IST001907 'Dynamically Evolving Large Scale Information Systems". This work was completed while the first author was visiting the RIPE NCC. the Internet today, and that the transition to native IPv6 is occurring slowly; thus, we expect tunnels to continue to play an important role in IPv6 networks, as IPv4 network infrastructure will remain widely deployed for many years.

Tunnel discovery is the process of automatically detecting tunnels and determining their endpoints. Similarly to other network discovery problems, its importance derives from the need for up-to-date information about network topology, and from the impact that topology is known to have on crucial aspects of network behavior, such as the dynamics of routing protocols [11], the scalability of multicast [12], the efficacy of denial-of-service countermeasures [13], [14], and other aspects of protocol performance [15].

From a practical perspective, the ability to discover tunnels can be useful in several scenarios. One example is troubleshooting: if a link in the tunnel's path fails, the tunnel fails, and IPv6 diagnostic tools such as traceroute 6 will not reveal the source of the problem. The ability to determine that the failed link is in a tunnel, and possibly perform an IPv4 traceroute between the tunnel endpoints, would provide great help in such cases. Secondly, tunnels are often used as an interim solution until native IPv6 infrastructure is in place. Tunnel detection techniques provide the means to follow the evolution of the IPv6 Internet from its origin as a completely tunneled network towards a completely native network, and determine how much has to be done to complete the migration to native IPv6. They can also provide insights into the structure of the network itself: for example, as the cost of a tunnel is much lower than that of a native link, predominantly tunneled regions may be more densely interconnected than native regions. The knowledge of these properties will aid the development of realistic IPv6 topology generators. Finally, tunnels offer lower performance than native links and are often used as backup paths in case of problems; the knowledge of whether a particular route contains a tunnel would allow routing protocols or network operators to prefer native routes. This is useful for Internet service providers and content delivery operators who wish to maximize the quality of service they provide.

Much has been written on the topic of IP topology discovery, which is usually performed by interacting with the network using probing packets [16]-[18] or through the observation of routing information, notably BGP tables [19], bridge forwarding tables [20], or IGP routing tables obtained via SNMP [21]. The combination of these approaches and the use of advanced techniques has led to the development of tools which achieve very good results in relatively little 


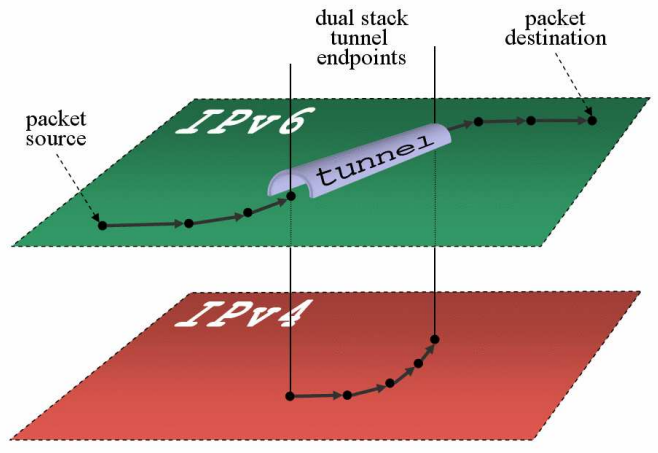

Fig. 1. An IPv6 in IPv4 tunnel is seen as a single hop at the IPv6 layer, but IPv6 packets are encapsulated and sent as the payload of IPv4 packets between the tunnel endpoints.

time [17], [19], [21]. However, tunnel discovery differs from other types of network discovery in that a tunneled network is made up of two distinct network layer topologies that interact, and the resulting network is thus a complex "overlay" of two forwarding planes (Figure 1), whose topology cannot be deduced simply by applying known methods to explore each plane separately; to do so, for example, would mean to completely ignore the path followed by the encapsulated packets in the encapsulating plane, which is clearly unsatisfactory. Further difficulties are caused by the fact that tunnels are transparent to the encapsulated network, appearing to be an ordinary point-to-point link while in reality they may span any number of links in the encapsulating network. The impact of tunnel discovery is also potentially more significant than that of other types of topology discovery because tunnels are more dynamic than physical links (for example, they can be automatically created using tunnel brokers [22] or specific tunnel setup protocols [23]) and because they can undermine both performance, as a single tunnel may hide a potentially long and inefficient path in the encapsulating network, and security, as our techniques based on IP spoofing clearly illustrate.

A possible approach to the tunnel discovery problem is the use of SNMP queries to obtain information directly from the nodes involved. This method is impractical, however: not only does it require administrative access to network equipment, and so cannot be used to discover tunnels in the Internet at large, but the required MIBs are not yet finalized [24], and the specific tunnel MIB is very rarely implemented. Another method was outlined in [25], which follows the approach of defining a new protocol and thus does not apply to existing infrastructure; furthermore, as it envisages authentication mechanisms, it suffers from the same drawbacks as the use of SNMP. In this paper, we discuss methodologies for tunnel discovery that do not require administrative access to the network and thus may be applied to the Internet at large. Our main contributions are the following:

- We introduce techniques to infer the existence of IPv6in-IPv4 tunnels, confirm the existence of inferred tunnels, and collect information about tunnel endpoints. We show how a tunnel, once discovered, can be used as a "vantage point" to launch third-party tunnel-discovery explorations.

- We describe Tunneltrace, a tool which uses our techniques to detect tunnels between a vantage point and a destination.

- We validate the techniques through wide experimentation, first on the 6bone tunneled network [30], then by observing the IPv6 Internet from hosts in native networks in Italy, the Netherlands and Japan, and from the test boxes deployed worldwide by the RIPE NCC as part of the Test Traffic Measurements Service [34]. As a byproduct of our experimentation, we are able to assess to what extent information in the 6bone registry is coherent with the actual network topology.

- Finally, we provide the first experimental results on the current distribution of tunnels in the Internet. Our data show that tunnels are very common, the percentage of native IPv6 connectivity is still low, and that the migration to native IPv6 is progressing, albeit slowly.

The paper is organized as follows: Section II briefly provides the basic definitions and notations used both in Section III, which introduces and formally describes our tunnel discovery techniques, and in Section IV, which describes Tunneltrace. Section V describes our experimentation and discusses our results. In Section VI we briefly address security issues introduced by tunnels that the development of our techniques has led us to discover. We conclude in Section VII.

\section{PReliminary definitions}

Our definitions of node, link and interface are consistent with the IPv6 specifications [26]: a node is a device implementing IPv6, a link is a communication medium, offered by an underlying link-layer (or, in the case of tunnels, networklayer) protocol, over which the IPv6 protocol may transmit packets, and an interface is a node's attachment to a link. A point-to-point link is a link to which exactly two interfaces are connected. A dual stack interface is an interface on which both IPv4 and IPv6 are enabled. We further (loosely) define a routable interface as an interface whose IPv6 address belongs to a prefix which exists in the global routing table and can thus be reached by any host on the network.

An IPv6-in-IPv4 tunnel, $T=\langle A, B\rangle$, is a point-to-point link between two dual stack interfaces $A$ (the tunnel source) and $B$ (the tunnel destination). We denote respectively with $A_{4}$ and $B_{4}$ and with $A_{6}$ and $B_{6}$ the IPv4 and IPv6 addresses of $A$ and $B$, and we represent bidirectional tunnels as two tunnels with the same endpoints in inverted order; thus, if a tunnel $T=\langle A, B\rangle$ is bidirectional, then $T^{\prime}=\langle B, A\rangle$ also exists. A tunnel operates as follows: when an IPv6 packet is sent through the tunnel from $A$ to $B$, the source node creates an IPv4 packet with source addresses $A_{4}$ and destination address $B_{4}$ whose payload is the IPv6 packet. Except in the case of GRE or Teredo tunnels, no extra headers are added and the packet is marked as encapsulating an IPv6 packet by setting the IPv4 Protocol field to 41 . The IPv4 packet is then sent to $B$ over the IPv4 network; when the destination node receives the packet, it examines the IPv4 source address to check whether 
it corresponds to a known tunnel, and if so, it decapsulates the packet and processes it normally, as if it had arrived on any other IPv6 interface. If the IPv6 packet is then forwarded, the Hop Limit field in the header is decremented by 1; thus, IPv6-in-IPv4 tunnels are "single-hop", that is, they appear to the IPv6 network as a single point-to-point link which hides the complexity of the underlying IPv4 network [3].

In the rest of the paper, we shall denote a packet with a pair of square brackets enclosing a source address, a destination address, and other important features of the packet itself. For example, an ICMPv6 echo request message from address $X_{6}$ to address $Y_{6}$ is written $\left[X_{6} Y_{6}\right.$ echo-request]. Packet encapsulation is described by recursively using square brackets: if the aforementioned IPv6 packet were encapsulated in an IPv4 packet, it would be written $\left[A_{4} B_{4}\left[X_{6} Y_{6}\right.\right.$ echo-request $]$. To denote the interface that originates or receives a packet, we prepend the packet with the interface followed by a colon or append to the packet the interface a colon followed by the interface, thus $X:\left[X_{6} Y_{6}\right.$ echo-request $]: Y$ is a packet sent by interface $X$ and received by interface $Y$. Finally, if the reception of a packet causes a node to emit another packet, we indicate this with the symbol $\triangleright$. For example, if an echo request packet causes a node to reply with an echo reply packet, we write $\left[X_{6} Y_{6}\right.$ echo-request $] \triangleright\left[Y_{6} X_{6}\right.$ echo-reply $]$.

\section{TUNNEL DISCOVERY METHODS}

This section presents a number of techniques we have developed to tackle the tunnel discovery problem. Depending on their objective, they may be divided into: (i) techniques to infer the existence of tunnels, (ii) techniques to confirm their existence, (iii) techniques to collect information about their endpoints, and (iv) techniques which allow a host to interact with the network as if it were located in a different place to the one in which it is actually located (we name these third party exploration techniques). They may further be characterized according to their mode of operation: some query known sources of information, others interact with the network and observe the results, performing what we may refer to as "active probing". Each technique is a suitable combination of the following basic methods:

Path MTU discovery The Maximum Transmit Unit (MTU) of a link is the maximum size of a packet that may be transmitted through the link, and the path MTU between two interfaces $X$ and $Y$ is the minimum MTU of the links composing the path between $X$ and $Y$. Path MTU discovery [27] is a method that allows a node to determine the path MTU between one of its interfaces and another interface on the network, thus obtaining information on the MTUs of the intervening links. The presence on the path of certain MTU values may suggest the presence of a tunnel.

DNS lookups The Domain Name System is used to map IPv4 and IPv6 addresses to hostnames and vice versa. Often the IPv4 and IPv6 addresses of an interface have the same name, and since tunnel interfaces are dual stack, DNS lookups can provide information about tunnel endpoints. DNS queries can also be used to determine whether an interface is dual stack.
TABLE I

CLASSIFICATION OF TUNNEL DISCOVERY TECHNIQUES

\begin{tabular}{|c||l||c|c|c|c|}
\hline$\#$ & Rule & $\begin{array}{c}\text { Infer } \\
\text { existence }\end{array}$ & $\begin{array}{c}\text { Confirm } \\
\text { existence }\end{array}$ & $\begin{array}{c}\text { Collect } \\
\text { information }\end{array}$ & $\begin{array}{c}\text { Third-party } \\
\text { exploration }\end{array}$ \\
\hline \hline 1 & MTU & $\times$ & & & \\
\hline 2 & DNS & $\times$ & & $\times$ & \\
\hline 3 & $\begin{array}{l}\text { Packet } \\
\text { injection }\end{array}$ & & $\times$ & & $\times$ \\
\hline 4 & $\begin{array}{l}\text { Fragment } \\
\text { injection }\end{array}$ & & $\times$ & & $\times$ \\
\hline 5 & $\begin{array}{l}\text { Injected } \\
\text { ping }\end{array}$ & & $\times$ & & \\
\hline 6 & $\begin{array}{l}\text { Dying } \\
\text { packet }\end{array}$ & & $\times$ & $\times$ & \\
\hline 7 & $\begin{array}{l}\text { Ping-pong } \\
\text { packet }\end{array}$ & & & $\times$ & \\
\hline 8 & $\begin{array}{l}\text { Bouncing } \\
\text { packet }\end{array}$ & & & $\times$ & \\
\hline
\end{tabular}

IP spoofing Because IPv6-in-IPv4 tunnels do not use any form of authentication, a tunnel destination will accept an encapsulated packet sent by any node as long as the source IPv4 address of the packet is the IPv4 address of the tunnel source. This allows any node to cause the tunnel endpoint to emit arbitrary IPv6 packets by encapsulating them in IPv4 packets with spoofed source addresses, and is the basis for our third-party discovery techniques.

Hop Limit manipulation The Hop limit field in the IPv6 header specifies the maximum number of routers a packet may pass through. When a router receives a packet with the Hop Limit field equal to 1, it discards it and sends the packet's source an ICMPv6 error message whose source address is the address of the interface on which it received the packet. This behavior may be exploited to discover the IPv6 address of an interface.

IPv6 Routing header While source routing is prohibited in the majority of IPv4 networks, many IPv6 routers honor the IPv6 Routing header, which permits a host sending a packet to specify a list of nodes that the packet is to pass through. Combined with Hop Limit manipulation, the Routing header can be useful for determining the addresses of point-to-point interfaces and tunnel interfaces in particular.

The remainder of this section is devoted to a formal presentation of the main techniques we have devised. Each technique is expressed by means of a formal rule, which is identified by a number and by a short name. Table I classifies the rules according to their objective. Although each rule is expressed by means of an implication, the validity of the implication is not absolute, and in real-world conditions a rule may fail to apply due to nonstandard behavior, misconfiguration, or unexpected and uncommon network topologies. Data on the applicability of the rules to real-world networks will be provided in Section $\mathrm{V}$.

Rule 1 (MTU): Consider the sequence of links that make up the path between some interface $X$ and some other interface $Y$. We may think of each link as a point-to-point link, because each packet that traverses a link is sent by exactly one of the interfaces on the link and is received by exactly one of the 


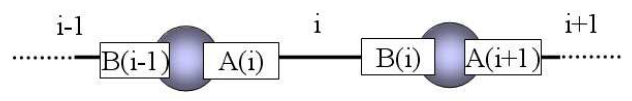

Fig. 2. A link $i$ in a path with its associated interfaces $A(i)$ and $B(i)$.

interfaces on the link ${ }^{1}$. Thus, if we number the links in the path progressively starting from 1 , for each we may define a source interface $A(i)$ and a destination interface $B(i)$, which have IPv6 addresses $A_{6}(i)$ and $B_{6}(i)$ (Figure 2).

Let $M T U(i)$ be the MTU of link $i$. If we can send packets from $X$, then we may use Path MTU discovery [27] to determine, for each link, the value

$$
\operatorname{PMTU}(i)=\min \{\operatorname{MTU}(i), \operatorname{PMTU}(i-1)\}
$$

where $P M T U(1)=M T U(1)$. Because of encapsulation, the MTU of the tunnel is lower than that of the underlying IPv4 network by a fixed amount depending on the tunnel type: 20 bytes for IPv6-in-IPv4 tunnels, 24 or 28 for GRE [10] tunnels. The most common MTU value on the IPv4 Internet today is 1500 bytes, so these tunnels will almost always have MTUs of 1480 and 1476 (or 1472) bytes respectively. Finally, many tunnel interfaces (notably on BSD systems) use a default MTU of 1280 bytes. Hence, if we consider two consecutive links $i-1$ and $i$ on the path, we may write:

$$
\begin{gathered}
\operatorname{PMTU}(i)<\operatorname{PMTU}(i-1) \wedge \\
\operatorname{PMTU}(i) \in\{1480,1476,1472,1280\} \Rightarrow \operatorname{Tunnel}(A(i), B(i))
\end{gathered}
$$

where Tunnel $(A(i), B(i))$ means that there is a tunnel between $A(i)$ and $B(i)$. Of course, if the tunnel is entirely contained in a portion of the IPv4 Internet where the MTU of all the links is higher than 1500 , this rule may fail to detect a tunnel. It may also wrongly detect a link as a tunnel if the link is manually configured to have an MTU equal to these values. This may be particularly common in the case of 1280 bytes, which is the minimum MTU permitted by the IPv6 specifications. The MTU rule is confirmed by experience in all the IPv6 networks on which we tested; note, however, that it will only find a tunnel if its MTU is lower than the MTU of all previous links in the path, and thus cannot detect two or more tunnels in the same path unless their MTUs are different.

Rule 2 (DNS): We represent DNS lookups with a function, Name(), that takes an IPv4 or IPv6 address and returns the corresponding DNS name, and two functions, $A d d r_{4}()$ and $A d d r_{6}()$, that take a DNS name and provide the corresponding IPv4 or IPv6 address. If a name of an interface $X$ has both an IPv6 and an IPv4 address, we may presume that it is dual stack and that the two addresses are its IPv4 and IPv6 address. Thus, we may write:

$$
\begin{aligned}
& \exists \alpha \mid \alpha=\operatorname{Addr}_{4}\left(\operatorname{Name}\left(X_{6}\right)\right) \Rightarrow \operatorname{DualStack}(X) \wedge X_{4}=\alpha \\
& \exists \beta \mid \beta=\operatorname{Addr}_{6}\left(\operatorname{Name}\left(X_{4}\right)\right) \Rightarrow \operatorname{DualStack}(X) \wedge X_{6}=\beta
\end{aligned}
$$

Rule 3 (Packet injection): Given two IPv4 addresses $A_{4}$ and $B_{4}$, if there is a tunnel between $A$ and $B$, it is possible

\footnotetext{
${ }^{1}$ This is usually the case for all packets, but load-balancing mechanisms or policy routing may cause behavior that varies from packet to packet.
}

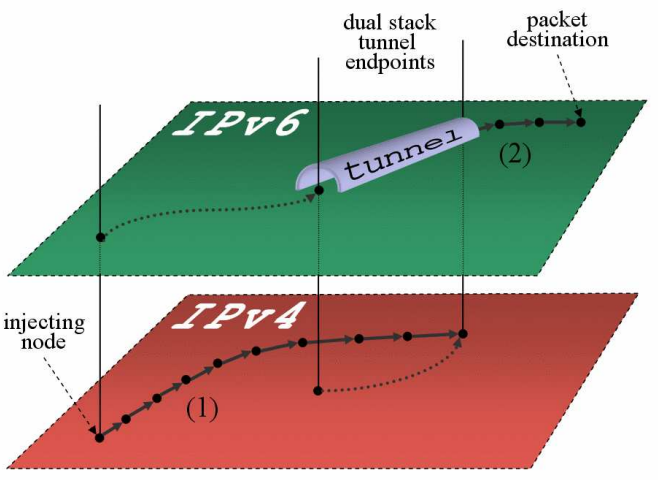

Fig. 3. Packet injection: A spoofed IPv4 packet is sent to a tunnel endpoint (1), is processed as if it had been sent by the other endpoint, and is forwarded to its destination (2).

to cause an arbitrary (though limited in size) IPv6 packet to enter the IPv6 network at interface $B$. This is done by sending, from any interface $Z$, an IPv6 packet encapsulated in an IPv4 packet with source and destination addresses $A_{4}$ and $B_{4}$. Because its source address is $A_{4}$, when the packet arrives at $B$ it will be recognized as arriving from the tunnel and will be decapsulated and processed as if it had been sent by $A$ (see Figure 3(a)). Formally, we may write:

$$
\begin{gathered}
\text { Tunnel }(A, B) \Rightarrow \\
Z:\left[A_{4} B_{4}\left[X_{6} Y_{6} \text { payload }\right]\right] \triangleright\left[X_{6} Y_{6} \text { payload }\right]: B
\end{gathered}
$$

where the payload of the two IPv6 packets is the same. This technique may be used to "inject" an arbitrary IPv6 packet, up to the maximum size permitted by the MTU of the underlying IPv4 network minus the size of the IPv4 header, into the IPv6 network at interface $B$. We refer to this technique as packet injection and to $Z$ as the injecting interface. Note that the packet, although sent by $Z$, actually enters the IPv6 network at interface $B$, and, as far as the IPv6 network is concerned, is simply a packet originated by a node on the same link as $B$. This rule allows the injecting host to send packets as if it were physically located on the same link as $B$, and if the node $r$ to which $B$ belongs is a router, the injected packet will be forwarded as normal towards $X$ as if it had been sent by $r$ itself. We then say that $r$ is a vantage point. Thanks to this rule, a single host in a single location may interact with and explore the network as if it were located simultaneously in all the vantage points it is aware of. Note, however, that because it depends on IP spoofing, both this rule and Rules 4, 5, 6 and 7 , which depend on it, will not work if the network in which $B$ is located makes use of IPv4 ingress filtering.

Rule 4 (Fragment injection): IPv6 packets injected using the packet injection technique described in Rule 3 are limited in size to the MTU of the underlying IPv4 network minus the size of the encapsulating headers. However, it is possible to inject a larger packet by fragmenting the IPv4 packet which encapsulates it. For example, suppose the packet $\left[A_{4} B_{4}\left[X_{6} Y_{6}\right.\right.$ payload $\left.]\right]$ is fragmented by the IPv4 network into two IPv4 packets $f_{1}$ and $f_{2}$. Upon arrival at $B$, the packet will be reassembled (resulting in an IPv4 packet larger than the 


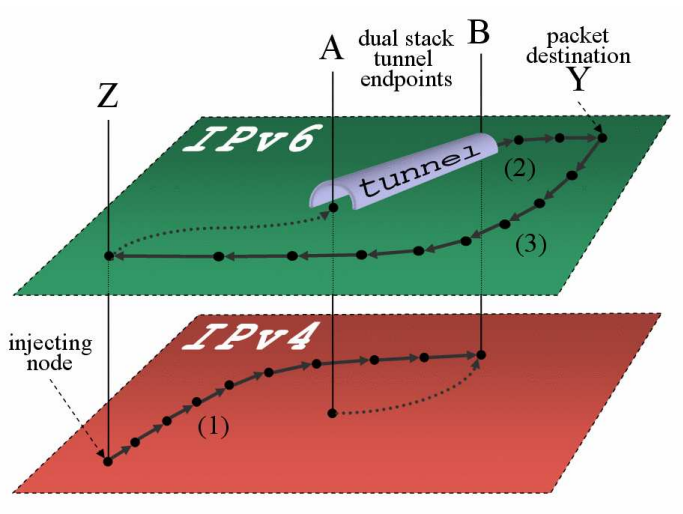

(a)

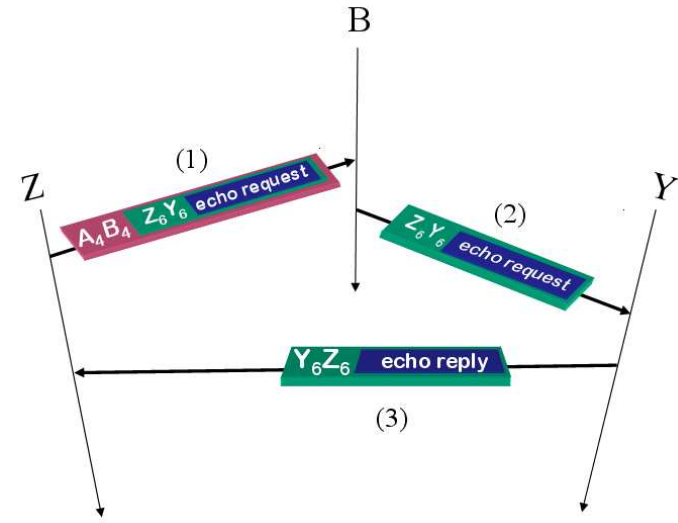

(b)

Fig. 4. Injected ping: an IPv6 echo-request packet is injected into a tunnel (1) and forwarded to its IPv6 destination address (2). The destination replies with an IPv6 echo-reply addressed to the source address of the encapsulated packet (3). Figure (a) shows the role of the tunnel, while Figure (b) shows the succession of events (vertical axes) and the relationship between the two protocols.

MTU of the IPv4 network) and will be processed according to Rule 3. So, we may write:

$$
\begin{gathered}
\text { Tunnel }(A, B) \wedge f_{1} \prec f_{2}=\left[A_{4} B_{4}\left[X_{6} Y_{6} \text { payload }\right]\right] \Rightarrow \\
Z: f_{1} \wedge Z: f_{2} \triangleright\left[X_{6} Y_{6} \text { payload }\right]: B
\end{gathered}
$$

where $f_{1}<f_{2}$ indicates the packet reassembled from the two fragments. This rule permits a host to use any vantage point $r$ to inject IPv6 packets of arbitrary size from $r$ as if it had a direct native connection to $r$. It is particularly useful in the search for tunnels: for example, by combining this rule with Rule 1, we may perform Path MTU discovery from the node having interface $B$.

Rule 5 (Injected ping): Given two IPv4 addresses $A_{4}$ and $B_{4}$, it is possible to determine whether there is a tunnel $T=\langle A, B\rangle$ by applying Rule 3 , with $X_{6}=Z_{6}$, to inject an echo request packet addressed to any routable interface $Y$ (Figure 4). The packet will arrive at interface $B$, and if there is no tunnel between $A$ and $B$, it will be discarded. Otherwise, it will be forwarded to its destination $Y$, which will reply with an echo reply message addressed to $Z_{6}$. If the injecting host receives a reply, it can conclude that there is a tunnel between $A$ and $B$. More formally, we may write:

$$
\begin{gathered}
Z:\left[A_{4} B_{4}\left[Z_{6} Y_{6} \text { echo-request }\right]\right] \triangleright\left[Y_{6} Z_{6} \text { echo-reply }\right]: Z \Rightarrow \\
\text { Tunnel }(A, B)
\end{gathered}
$$

Rule 6 (Dying packet): Given a tunnel $T=\langle A, B\rangle$, it is possible to determine the IPv6 address $B_{6}$ of the tunnel destination by injecting a packet with the IPv6 Hop Limit field set to 1. Because IPv6-in-IPv4 tunnels are modeled as "single-hop", the packet will appear at interface $B$ without ever having been processed by an IPv6 router, and thus with the contents of the Hop Limit field intact. Upon arrival at interface $B$, however, the Hop Limit of the packet will be decremented to zero. The resulting "time exceeded" message will arrive at $Z$ and the injecting host may determine $B_{6}$ by examining its source address. Stating this in terms of a rule, we have:

$$
Z:\left[A_{4} B_{4}\left[Z_{6} X_{6} \mathrm{HL}=1\right]\right] \triangleright\left[Y_{6} Z_{6} \text { time exceeded }\right]: Z \Rightarrow
$$

$$
B_{6}=Y_{6}
$$

If the tunnel is bidirectional, it is possible to determine the IPv6 address of the other endpoint simply by exchanging $A_{4}$ and $B_{4}$.

Rule 7 (Ping-pong packet): Rule 6 does not allow us to determine the IPv6 address $A_{6}$ of the tunnel source if the tunnel is not bidirectional. However, it is frequently possible to determine it by other means. Suppose the tunnel has an IPv6 prefix $T$ associated with it. Any IPv6 address in $T$ will be routed towards the tunnel, and each tunnel endpoint will route through the tunnel all addresses in $T$ except its own. Thus, if we use Rule 5 to inject an echo request packet with a Hop Limit of 2 and destination address $X_{6}$ in $T$ but not equal to $B_{6}$, the packet will reach $B$ and be sent back through the tunnel to $A$. If $X_{6}=A_{6}$, then the injecting host will receive an echo reply. Otherwise ${ }^{2}$, it will receive a "time exceeded" message with source address $A_{6}$. In both cases, it obtains $A_{6}$.

It is simple to determine a suitable value for $X_{6}$ : the length of $T$ must be at most 127 , because otherwise $A_{6}$ and $B_{6}$ cannot both be in $T$. So, whatever the length of $T, B_{6} \pm 1$ (where the sign depends on whether $B_{6}$ is even or odd) is always in $T$. So we may write:

$$
\begin{array}{r}
Z:\left[A _ { 4 } B _ { 4 } \left[Z_{6} X_{6}\right.\right. \text { echo-request,HL=2]] } \\
\triangleright\left[X_{6} Z_{6} \text { echo-reply }\right]: Z \\
Z:\left[A _ { 4 } B _ { 4 } \left[Z_{6} X_{6}\right.\right. \text { echo-request,HL=2]] } \\
\triangleright\left[Y_{6} Z_{6} \text { time exceeded }\right]: Z
\end{array} \quad \Rightarrow \quad A_{6}=X_{6}
$$

where

$$
X_{6}= \begin{cases}B_{6}+1, & \text { if } B_{6} \text { is even } \\ B_{6}-1, & \text { if } B_{6} \text { is odd }\end{cases}
$$

Note that if the tunnel interfaces are unnumbered, $A_{6}$ and $B_{6}$ are not on the same subnet and this rule does not apply.

\footnotetext{
${ }^{2}$ If subnet-router anycast addresses are used, $X_{6}$ may belong to the same router as $B_{6}$. However, the injecting host may determine if that is the case simply by sending $\left[A_{4} B_{4}\left[Z_{6} X_{6}\right.\right.$ echo-request,HL=1] and seeing if $B$ responds with an echo reply or a time exceeded. If it responds with an echo reply, then subnet-router anycast is active, so the subnet is at least a /126. The injecting host can then choose $X_{6}$ as another address in the subnet.
} 
Rule 8 (Bouncing packet): Consider the path from some interface $Z$ to some other interface $W$. An IPv6 traceroute from $Z$ to $W$ allows us to determine the sequence $B_{6}(i)$, where $i=1 \ldots n$ is the $\mathrm{i}$-th link in the path, but it does not allow us to determine $A_{6}(i)$ for any $i$. However, $Z$ may use the IPv6 Routing header to send a packet to $B(i)$ which is routed back towards itself; if the Hop Limit $H L$ of this packet is set to the appropriate value, the packet will expire on interface $A(i)$ and $Z$ will receive a "time exceeded" message with source address $A_{6}(i)$. The required value of $H L$ is not necessarily $i+1$, as the path taken by a packet $\left[Z_{6} B_{6}(i)\right]$ may not be a subpath of the path taken by a packet $\left[Z_{6} W_{6}\right]$. However, $H L$ may be determined by adding one to the number $x$ of hops between $Z$ and $B(i)$, which can be obtained, for example, by running a traceroute from $Z$ to $B_{6}(i)$.

In the presence of asymmetric routing, this may not provide $A_{6}(i)$, because the path from $B(i)$ to $Z$ may not be the same as the path from $Z$ to $B(i)$. This problem may be partially overcome by setting the packet's destination not to $Z_{6}$ but to a previous hop on the path, to reduce the effects of route asymmetry. Because the ICMPv6 specification requires [28, section 2.2] that if the packet is sent to $B_{6}(i-1)$, the source address of the error message must be $B_{6}(i-1)$ and not $A_{6}(i)$, $Z$ may set the destination address to $B_{6}(i-2)^{3}$. More formally,

$$
\begin{gathered}
Z:\left[Z_{6} B_{6}(i) B_{6}(i-2) \mathrm{HL}=x+1\right] \triangleright\left[Y_{6} Z_{6} \text { time-exceeded }\right]: Z \\
\Rightarrow A_{6}(i)=Y_{6}
\end{gathered}
$$

where $\left[Z_{6} B_{6}(i) B_{6}(i-2)\right]$ indicates a packet source routed through $B_{6}(i)$ with destination $B_{6}(i-2)$. While this rule applies to any link, not only to tunnels, it is particularly useful when combined with Rule 2 to determine tunnel endpoints given path information; if the IPv4 addresses of the tunnel endpoints are known, then Rules 6 and 7 are more effective.

\section{A TUNNEL DISCOVERY TOOL}

In this section we describe Tunneltrace, a tunnel discovery tool we have developed to test the techniques introduced in Section III. Although Tunneltrace is not intended to be the main contribution of our work, which we believe lies in the techniques themselves, we present it here as an example of their application. Tunneltrace attempts to detect, and collect information about, tunnels in the path between the exploring host and a user-specified destination. Of course, by applying Rule 4, in principle it is possible, given a sufficient number of vantage points, to find tunnels in the entire network.

The strategy followed by Tunneltrace is simple: perform a traceroute to the destination node, and for each link $i$ attempt to discover if it is a tunnel. If it is, attempt to discover the IPv4 addresses of the endpoints, confirm the tunnel's presence, and use it as a vantage point to explore the rest of the path.

Specifically, for each hop in the traceroute $B_{6}(i)$, Tunneltrace first applies Rule 1 to determine whether

\footnotetext{
${ }^{3}$ This may still provide incorrect results, because the asymmetry may be located between $B(i)$ and $B(i-2)$. If greater accuracy is desired, the inferred value of $A_{6}(i)$ can be compared with $B_{6}(i)$ and accepted only if it is on the same subnet; however, this will cause false negatives for unnumbered links.
}

link $i$ is a tunnel. If so, it attempts to obtain information about its endpoints in the following way: first, it attempts to obtain the IPv6 address of the previous hop's sending interface, $A_{6}(i)$, using Rule 8; then, it uses Rule 2 to attempt to obtain $A_{4}(i)$ and $B_{4}(i)$, and if it succeeds, it attempts to confirm the presence of the tunnel using Rule 5; finally, it verifies the information collected by using Rules 6 and 7. If the tunnel is confirmed, it is used as a vantage point to explore the rest of the path.

If Rule 2 does not provide enough information to use the tunnel as a vantage point, Tunneltrace combines it with heuristics on DNS names, attempting to perform piecewise matching as proposed in [29], and, if the name contains strings such as "v6-", "ip6.", or "ipv6.", repeating the DNS lookup after removing them.

Tunneltrace also examines names looking for strings that suggest the presence of tunnels (such as "tunnel" or "tu"), queries the 6bone registry to check whether the node is a known tunnel endpoint, and performs AS lookups: if the IPv4 address of a node is in a different AS as its IPv6 address, or if the hops before and after the node are in different ASes than the node itself, the node may be the endpoint of an interdomain tunnel. In all these cases, Tunneltrace reports that a tunnel might be present.

For each hop, Tunneltrace also outputs information such as the IPv6 address (and DNS name and AS number) of the answering interface $B_{6}(i)$ and whether the interface is dual stack. It also provides this information about the sending interfaces $A(i)$.

\section{EXPERIMENTAL RESULTS}

The 6bone experimental IPv6 network [30] provides a useful testbed for our work, as data on tunnels is publicly available in the 6bone registry. Thus, applying our techniques to the 6bone may both (i) allow us to verify the validity of our techniques, and (ii) use our techniques to check the accuracy of the information in the registry itself. We used the tunnel data available in the 6bone registry in various ways. Firstly, we checked it for consistency, using DNS lookups and packet injection to determine how many tunnels in the tunnel database actually exist. Secondly, we used it as a large list of tunnels against which to check the validity of our tunnel discovery techniques. Finally, we used the list of existing tunnels as vantage points from which to search for tunnels in the IPv6 Internet at large.

\section{A. Status of the 6bone registry}

We applied our tunnel confirmation methods to the 6bone registry to determine whether the information on tunnels it contains is accurate and up to date and to determine whether the quality of the information varies over time. For every tunnel, the registry contains the DNS names or IPv4 addresses of the tunnel endpoints, along with other information. We process one tunnel at a time, and attempt to resolve the DNS hostnames of the tunnel endpoints to IPv4 addresses. If both names can be translated to IP addresses, we use Rule 5 to 
TABLE II

STATUS OF TUNNELS IN THE 6BONE REGISTRY

\begin{tabular}{|c||c|c|c|c|c|}
\hline Date & Total Tunnels & Up & Down & $\begin{array}{c}\text { One endpoint } \\
\text { unknown to DNS }\end{array}$ & $\begin{array}{c}\text { Both endpoints } \\
\text { unknown to DNS }\end{array}$ \\
\hline \hline $2003-06-13$ & 4334 & 998 & 1479 & 1328 & 529 \\
\hline $2003-06-23$ & 4319 & 1058 & 1394 & 1333 & 534 \\
\hline $2003-07-18$ & 4202 & 998 & 1322 & 1342 & 540 \\
\hline $2003-08-07$ & 4197 & 1046 & 1345 & 1316 & 490 \\
\hline \hline $2004-02-27$ & 4280 & 964 & 1310 & 1397 & 609 \\
\hline
\end{tabular}

determine whether the tunnel is actually working. The results of our analysis are in Table II.

The results show that almost half of all tunnel records in the registry have invalid DNS names for one or both endpoints and therefore are either out of date or refer to tunnels that no longer exist. About a quarter of the records are working tunnels. The rest do not permit packet injection; while some of these may be GRE tunnels and/or have endpoints located in networks that employ ingress filtering, we expect most of them to be inactive: our MTU survey results indicate that GRE tunnels are much less common than IPv6-in-IPv4 tunnels, and as the majority of tunnels in the 6bone registry are interdomain tunnels, it is unlikely that ingress filtering has any significant impact on the results. Further study of these undecided cases would allow the development of a tool which could monitor all aspects of the quality of a tunnel registry.

To study variation over time, we carried out a number of observations over a two-month period, between June and August 2003, to observe short-term changes. Our results show that the data in the registry is fairly static, with a slow rate of change. Comparison with a further observation made approximately six months later, in February 2004, indicates that the the quality of the information stored in the registry is slowly decreasing: over this six month period, the percentage of working tunnels dropped from $24.9 \%$ to $22.5 \%$, and the percentage of tunnels for which one or both endpoints had an invalid DNS name rose from $43.0 \%$ to $46.9 \%$. This is consistent with the fact that the 6bone is being phased out [31].

\section{B. Rule validity data}

The large number of working tunnels provided by the 6bone registry allows us to validate our tunnel discovery techniques against known data: once a tunnel is confirmed using Rule 5, we may check the validity of Rules $2,4,6$, and 7 . Using the 2003-08-07 dataset, we checked whether these rules applied to the tunnels in the registry that we had confirmed to be working. Of a total sample of 1046 tunnels, we found that Rule 4 (Fragment Injection) applied to 999 tunnels (95.5\%) and Rule 6 (Dying packet) applied to 1013 tunnels (96.8\%). Rule 7 was tested only on tunnels that did not permit packet injection in both directions, because for these, Rule 6 is much more effective. Of 218 tunnels that were not bidirectional, Rule 7 applied to 151 (69.2\%). Together, Rules 6 and 7 allowed us to determine both IPv6 endpoints for 963 tunnels (92.1\%).

Rule 2 (DNS) was significantly less useful: of the 963 tunnels for which we knew the IPv6 addresses of both endpoints, it applied to one endpoint in 169 cases (17.5\%), and to both endpoints in only 6 cases $(0.6 \%)$. Though its utility is rather limited, we feel that as it is probably the most obvious of our techniques, our work would not be complete without discussing it and determining its degree of usefulness. Clearly, the techniques that make use of active probing produce significantly better results than can be obtained by querying online sources of information such as the 6bone registry or the DNS.

\section{MTU survey}

The large number of vantage points obtained from the 6bone registry allows us to use third-party exploration to evaluate the impact of tunnels in a sizable portion of the IPv6 Internet. Using a sample of 995 vantage points in 92 different Autonomous Systems (ASes; for comparison, the total number of ASes which announce IPv6 routes is approximately 450), we applied Rule 4 to perform Path MTU discovery from each vantage point to every prefix in the IPv6 routing table. By applying Rule 1, we may deduce which paths contain one or more tunnels and which are native. The results of our analysis, excluding connectivity errors, are in Table III.

TABLE III

PATH MTU VALUES FROM VANTAGE POINTS TO THE INTERNET AS OF August 2003

\begin{tabular}{|c|c|c|}
\hline MTU value & Number of paths & Percentage \\
\hline \hline 1480 & 150946 & $39.4 \%$ \\
\hline 1280 & 138358 & $36.1 \%$ \\
\hline 1476 & 44404 & $11.6 \%$ \\
\hline 1500 & 31525 & $8.2 \%$ \\
\hline 1428 & 13619 & $3.6 \%$ \\
\hline Other & 4104 & $1.1 \%$ \\
\hline Total & 382956 & $100.0 \%$ \\
\hline
\end{tabular}

We note that the most common MTU is 1480 bytes, that of an IPv6-in-IPv4 tunnel, followed by 1280, the minimum IPv6 MTU, which indicates that at least one link in the path has a MTU of 1280 bytes (possibly an IPv6-in-IPv4 tunnel on a BSD system). The paths with an MTU of 1476 are probably due to GRE tunnels, while the paths with a MTU of 1428 may be due to encapsulation of IPv6 in a L2TP VPN. Native paths (those with a MTU of 1500) make up only $8.2 \%$ of all the paths we surveyed. A relatively low percentage of native paths is to be expected, given the fact that our vantage points are tunnel endpoints, and each probably reaches some percentage of prefixes through the tunnel itself; nevertheless these results allow us to affirm that the percentage of native paths in the IPv6 Internet is still quite low. 


\section{Survey of "native" IPv6 networks: how native is native?}

So far we have observed the IPv6 Internet through vantage points that are tunnel endpoints. Since these may be located in portions of the network that are dense in tunnels, we conducted a survey from hosts inside three native IPv6 networks, one in the GARR [32] network in Italy (AS 137), one at the RIPE NCC in the Netherlands (AS 3333), and one at WIDE in Japan (AS 2500) [33], to discover how tunneled "native" networks really are. From each host we measured the Path MTU to every prefix in the global IPv6 BGP table and applied Rule 1 to determine whether the path to each prefix contained at least one tunnel. We found that of 443 prefixes in the BGP table at the time of the analysis, the GARR, RIPE NCC and WIDE networks respectively reached at least 275 (64.3\%), 305 $(68.8 \%)$ and $308(72.6 \%)$ through tunnels ${ }^{4}$

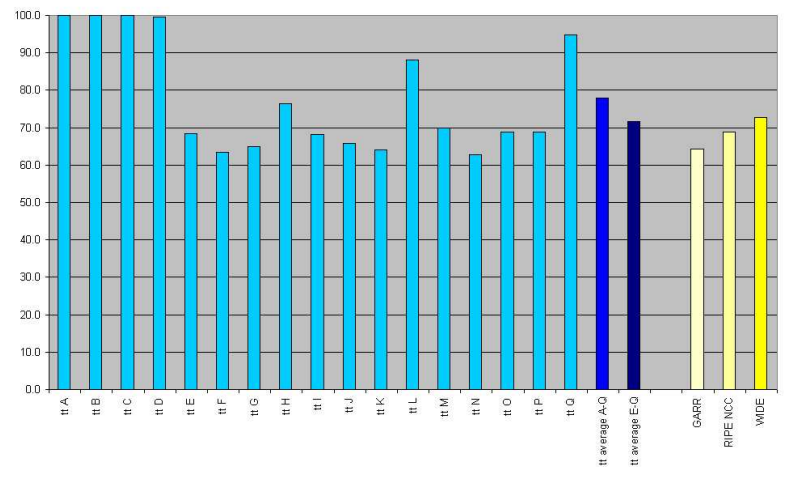

Fig. 5. Percentage of tunneled prefixes seen by TTM test-boxes and three native sites as of February 2004.

In order to obtain a more complete picture of the effect of tunnels on global IPv6 connectivity, we repeated the experiment from the "test-boxes" deployed worldwide by RIPE NCC as part of the Test Traffic Measurements service [34]. Over 100 test-boxes are currently active, of which about 20 have IPv6 connections. We repeated the tests three times, once in August 2003, once in January 2004, and once in February 2004; for each test, the same list of prefixes, obtained from a BGP router in AS 137 on the day of the test, was used for all the hosts. Due to differences in routing, not all test-boxes could reach all prefixes, so prefixes that were reported to be unreachable by a router within a test-box's own AS were not factored into the results. Also, test-boxes that could not reach at least $75 \%$ of the BGP prefixes in our list were excluded from the results so as not to skew the averages.

The February 2004 results are in Figure 5. As can be seen from the graph, four of the test-boxes reached $100 \%$ or almost $100 \%$ of the prefixes through tunnels, from which we deduce that they are located in networks which do not have a native IPv6 connection; the others reached between $62.8 \%$ and $94.8 \%$ of the prefixes through tunnels. The column labeled "tt average A-Q" provides an average for all 17 testboxes, while the column labeled " $t$ average E-Q" provides the

\footnotetext{
${ }^{4}$ Discrepancies between number of prefixes and percentages are due to the fact that not all these networks could reach the same number of prefixes, and unreachable prefixes were not counted in the results.
}

average of all natively connected test-boxes. The results show that global IPv6 connectivity still relies largely on tunnels, even when observed from a native IPv6 network. However, we note that there are non-trivial differences between the percentage of native destinations reached by the various testboxes, indicating that measurements such as these offer a good indication of the quality of an IPv6 network.

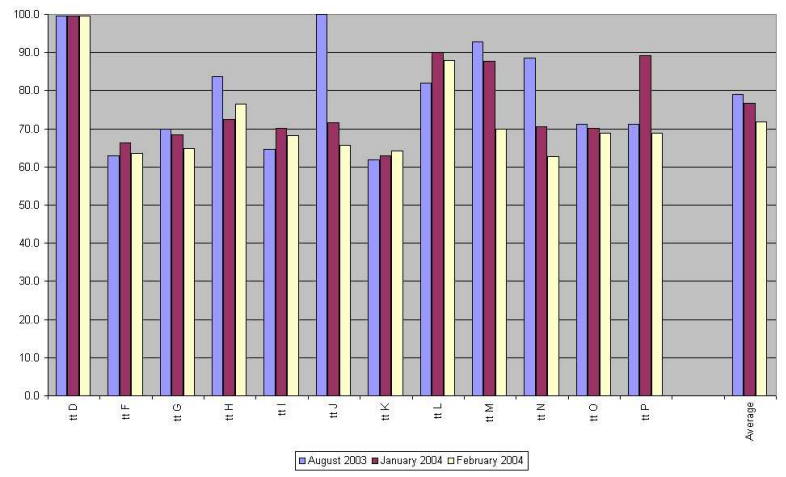

Fig. 6. Change in the percentage of tunneled prefixes seen by various test boxes over a period of six months.

As regards variation over time, Figure 6 shows the evolution of the percentage of prefixes reached through tunnels for the 12 test-boxes for which we have complete data sets (in August 2003, some test-boxes did not yet exist, and others did not have connectivity to IPv6 networks yet; in January 2004 a further test-box was unavailable due to technical problems). Our data indicate that the quality of the IPv6 network is slowly improving, as the average percentage of tunneled prefixes seen by these hosts decreased from $79.0 \%$ in August 2003 to 76.6\% in January and $71.7 \%$ in February 2004. Some of this variation is due to $\mathrm{tt} \mathrm{J}$ being changed from a tunneled connection to a native connection between August 2003 and January 2004 and to tt $\mathrm{P}$ experiencing non-optimal performance in January 2004, but even excluding these two test-boxes the percentages still decrease over time: $77.7 \%$ in August 2003, 75.8\% in January 2004, and $72.6 \%$ in February 2004. We believe that continued monitoring using our techniques would enable us to track the evolution of the IPv6 network towards global native connectivity and possibly provide insight into the processes that drive it, although we leave this for future work.

\section{SECURITY CONSIDERATIONS}

The development of our techniques, especially those related to third-party discovery, has led us to consider security issues inherent in IPv6-in-IPv4 tunnels. The fact that the IPv6 specifications do not define any type of authentication mechanism for tunnels except a check on the IPv4 packet's source address, which is easily circumvented by IP spoofing, is a flaw that may be exploited by a malicious user: as we have shown in Rule 3, an attacker may inject arbitrary IPv6 packets into the IPv6 network at a tunnel endpoint simply by by spoofing the IPv4 address of the other endpoint.

From the perspective of an attacker, packet injection is more attractive than IPv6 source address spoofing, since it 
has the potential to bypass IPv6 packet filters (by using IPv4) and because the attacker can use a real IPv6 source address and receive replies, thus allowing the establishment of TCP connections (which is not normally possible using IP spoofing). This method may thus be used to bypass firewalls and circumvent IPv6 ingress filtering if the tunnel endpoint is located behind them. An example is in Figure 7.

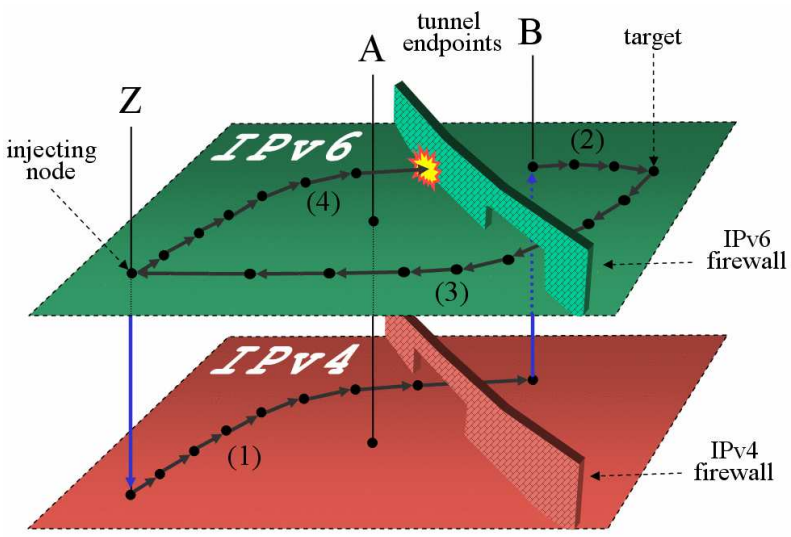

Fig. 7. If a tunnel, even one limited to a known IPv4 address, for example that of a trusted user for remote access, has an endpoint behind the IPv6 firewall, it allows any dual stack host $Z$ to inject IPv6 packets into the internal network, bypassing the firewall. If $Z$ 's packets were routed normally through the IPv6 Internet, they would be blocked by the IPv6 firewall (4), but if $Z$ encapsulates the packets and spoofs $A$ 's IPv4 address, the packets bypass the IPv6 firewall on the IPv4 plane (1) and reach the target (2). Firewalling on the IPv4 plane does not help, as the IPv4 firewall must be configured to allow IPv4 packets from the legitimate user.

The resulting security implications are similar to those of source routing, which is administratively prohibited in the majority of IPv4 networks; however, from the perspective of an attacker this method may be even more attractive than IPv6 source routing, because it cannot easily be guarded against without harming legitimate traffic and because the packets arrive at the tunnel endpoints with the IPv6 Hop Limit field left intact. This allows the attacker to spoof Neighbor Discovery messages, with possibly dangerous consequences.

IPv4 ingress filtering removes the problem by preventing IP spoofing, but this solution cannot easily be applied to interdomain tunnels such as those used in the 6bone. Thus, if interdomain tunnels are used, their endpoints should be kept outside firewalls, or different types of tunnels, such as GRE [10] or keyed GRE tunnels, should be used. The use of IPsec is also an obvious (but non-trivial) solution, as is, of course, the use of native links instead of tunnels.

\section{CONCLUSIONS}

We have introduced several techniques to infer the existence of IPv6-in-IPv4 tunnels, to confirm their existence, and to collect information about their endpoints, outlining a strategy for tunnel discovery along a path and showing how it is possible to use tunnels as "vantage points" to inject packets into the network at multiple locations, performing third-party exploration and scaling up the discovery process.

By applying our techniques to the 6bone registry, we were able to assess to what degree it is coherent with the actual state of the network, showing that almost half of the information on tunnels is out of date, but that about one quarter or more of the tunnels listed is still functioning. The information in the 6bone registry also allowed us to verify the validity of our techniques, showing that those which make use of active probing are very effective, providing results for over $90 \%$ of the tunnels in the registry we were able to make use of.

We used our techniques to provide the first experimental data on the presence of tunnels in the IPv6 Internet, by measuring the percentage of IPv6 prefixes reached through tunnels from the native GARR, RIPE NCC and WIDE networks and from the 20 IPv6-enabled test-boxes deployed worldwide by the RIPE NCC as part of the Test Traffic Measurements service. All the networks we tested reached less than $40 \%$ of IPv6 prefixes natively, showing that global IPv6 connectivity still relies largely on tunnels.

We also considered the security issues posed by IPv6in-IPv4 tunnels, which provide only a very weak form of authentication, touching on the possible threats that may arise and the countermeasures that may be taken.

\section{ACKNOWLEDGEMENTS}

We would like to thank Henk Uijterwaal from the RIPE NCC for providing us with the opportunity to perform our measurements from the RIPE NCC network and the TTM test-boxes. We would also like to thank Kenjiro Cho for his comments and help on MTU measurements from Japan and the WIDE Project [33] for allowing the use of its network infrastructure for this purpose.

\section{REFERENCES}

[1] R. Callon and D. Haskin, "RFC2185: Routing aspects of IPv6 transition," September 1997.

[2] D. Provan, "RFC1234: Tunneling IPX traffic through IP networks," June 1991.

[3] R. Gilligan and E. Nordmark, "Transition mechanisms for IPv6 hosts and routers," RFC 2893, Aug. 2000

[4] R. Woodburn and D. Mills, "RFC1241: Scheme for an internet encapsulation protocol: Version 1," July 1991.

[5] S. Kent and R. Atkinson, "Security architecture for the Internet Protocol," RFC 2401, Nov. 1998.

[6] B. Gleeson, A. Lin, J. Heinanen, G. Armitage, and A. Malis, "A Framework for IP Based Virtual Private Networks," RFC 2764, February 2000.

[7] B. Carpenter and K. Moore, "Connection of IPv6 domains via IPv4 clouds," RFC 3056, Feb. 2001.

[8] F. Templin, T. Gleeson, M. Talwar, and D. Thaler, "Intra-Site Automatic Tunnel Addressing Protocol (ISATAP)," Work in progress, Apr. 2002.

[9] C. Huitema, "Teredo: Tunneling IPv6 over UDP through NATs," Work in progress.

[10] S. Hanks, T. Li, D. Farinacci, and P. Traina, "RFC1701: Generic Routing Encapsulation (GRE)," October 1994.

[11] C. Labovitz, A. Ahuja, A. Bose, and F. Jahanian, "Delayed internet routing convergence." in ACM SIGCOMM 2000, September 2001.

[12] G. Philips, S. Shenker, and H. Tangmunarunkit, "Scaling of multicast trees: Comments on the Chuang-Sirbu scaling law," in ACM SIGCOMM 1999, August 1999.

[13] S. Savage, D. Wetherall, A. Karlin, and T. Anderson, "Practical network support for IP traceback," in Proc. ACM/SIGCOMM '00, Aug. 2000.

[14] K. Park and H. Lee, "On the effectiveness of route-based packet filtering for distributed DoS attack prevention in power-law internets," in Proc. ACM/SIGCOMM '01, Aug. 2001.

[15] P. Radoslavov, H. Tangmunarunkit, H. Yu, R. Govindan, S. Shenker, and D. Estrin, "On characterizing network topologies and analyzing their impact on protocol design," September 2000. 
[16] H. Burch and B. Cheswick, "Mapping the Internet," IEEE Computer, vol. 32, no. 4, pp. 97-98, April 1999.

[17] N. Spring, R. Mahajan, and D. Wetherall, "Measuring ISP topologies with rocketfuel," in Proc. ACM/SIGCOMM '02, Aug. 2002. [Online]. Available: citeseer.nj.nec.com/spring02measuring.html

[18] I. Astic and O. Festor, "A hierarchical topology discovery service for IPv6 networks," in IEEE/IFIP Network Operations and Management Symposium NOMS'2002., R. Stadler and M. Ulema, Eds., April 2002, pp. 497-510.

[19] G. Di Battista, F. Mariani, M. Patrignani, and M. Pizzonia, "Archives of BGP updates: Integration and visualization," in Proc. International Workshop on Inter-domain Performance and Simulation, Salzburg, Austria, Feb. 2003.

[20] Y. Bejerano, Y. Breitbart, M. Garofalakis, and R. Rastogi, "Physical topology discovery for large multi-subnet networks," in Proc. IEEE/INFOCOM '03, 2003.

[21] G. Barbagallo, "Polyphemus, a system for discovering and visualizing OSPF networks," http://www.dia.uniroma3.it/ polyph/.

[22] A. Durand, P. Fasano, I. Guardini, and D. Lento, "IPv6 tunnel broker," RFC 3053, Jan. 2001.

[23] M. Blanchet, "Tunnel setup protocol (tsp): A control protocol to setup IPv6 or IPv4 tunnels," Work in progress.
[24] B. Fenner, B. Haberman, J. Schoenwalder, and D. Thaler, "Management information base for the Internet Protocol (IP)," Work in progress.

[25] R. Bonica, K. Kompella, and D. Meyer, "Tracing requirements for generic tunnels," Work in progress.

[26] S. Deering and R. Hinden, "Internet Protocol, version 6 (IPv6) specification," RFC 2460, Dec. 1998.

[27] J. McCann, S. Deering, and J. Mogul, "Path MTU discovery for IP version 6," RFC 1981, Aug. 1996.

[28] A. Conta and S. Deering, "Internet control message protocol (ICMPv6) for the Internet Protocol version 6 (IPv6) specification," RFC 2463, Dec. 1998.

[29] V. N. Padmanabhan and L. Subramanian, "An investigation of geographic mapping techniques for internet hosts," 2001, p. 13. [Online]. Available: citeseer.nj.nec.com/padmanabhan01investigation.html

[30] R. Rockell and R. Fink, "RFC2772: 6Bone Backbone Routing Guidelines," February 2000.

[31] R. Fink and R. Hinden, "6bone (IPv6 Testing Address Allocation) Phaseout," Work in progress.

[32] "GARR - The Italian Academic and Research Network," http://www.garr.it/.

[33] "Widely Integrated Distributed Environment (WIDE) Project," http://www.wide.ad.jp/.

[34] “RIPE NCC Test Traffic Measurements," http://www.ripe.net/ttm/. 